\title{
Circuler pour travailler : les migrations économiques en Europe
}

Paru dans Boris Petric, Jean-François Gossiaux, Europa mon amour. 1989-2009 : Un rêve blessé, Paris, Autrement, 2009, pp. 193-206.

Swanie Potot,potot@unice.fr

\section{Introduction}

Dans l'imaginaire occidental, la chute du mur de Berlin est devenue le symbole de la réunification d'un continent artificiellement coupé en deux durant plusieurs décennies. Pourtant, si l'effondrement politique des régimes socialistes est accueilli très favorablement à l'ouest du continent, l'inclusion des populations est-européennes dans un vaste espace de libre circulation est reçue avec davantage de réticences.

A l'époque d'un antagonisme de blocs, les personnes qui réussissaient, sporadiquement, à s'échapper du «bloc de l'est » étaient accueillies à bras ouverts en Europe de l'Ouest et «l'Occident démocratique » honorait fidèlement sa réputation de terre d'asile. En rejoignant nos pays, souvent au péril de leur vie, ces réfugiés politiques soulignaient le bien-fondé du choix capitaliste. Leur faible nombre, garanti par l'érection des barrières dont l'Occident ne portait pas la responsabilité, évacuait toute menace d'invasion. Ces émigrés, souvent artistes, intellectuels ou sportifs de haut niveau profitaient de quelques privilèges réservés aux élites pour quitter leur pays, contribuant dès lors au rayonnement de la nation qui les accueillait.

Les évènements de 1989 marquent la fin de cet équilibre. En quelques mois, les émigrés du monde communiste se sont mués en des migrants économiques menaçant la quiétude des sociétés occidentales. Dès 1990, la peur de se voir envahis par des hordes de migrants venus de l'Est se répand dans les medias comme en politique. Pour autant, le rêve de construire une Europe commune au sein de laquelle marchandises et personnes pourraient circuler librement n'est pas abandonné. L'effacement progressif des frontières à l'intérieur (d'une partie seulement) de cette nouvelle Europe prendra plus de vingt années. En France, ce n'est qu'en 2008 que l'on ouvrira le marché du travail aux ressortissants de huit pays d'Europe Centrale et Orientale (PECO) ${ }^{1}$, laissant les autres dans l'attente ou dans la marge.

Le processus d'ouverture vers l'Est a été progressif. Dès les années 90, loin des grands discours, se sont tissés des liens, créées des niches d'emploi, organisés des réseaux

\footnotetext{
${ }^{1}$ Arrêté du 24 juin 2008 relatif à la délivrance, sans opposition de la situation de l'emploi, des autorisations de travail aux ressortissants des Etats de l'Union européenne soumis à des dispositions transitoires. Cet arrêté ne concerne pas la Roumanie et la Bulgarie, pourtant membres de l'UE depuis 2007.
} 
transnationaux entre les deux Europes. Cet espace de circulation s'est construit conjointement via des politiques migratoires basées sur la maitrise des flux et des pratiques informelles d'acteurs sociaux.

\section{Circulation touristique pour les uns}

Une première ouverture voit le jour en 1991. Les membres de l'espace Schengen ${ }^{2}$ ont supprimé l'obligation de visa pour des séjours touristiques inférieurs à trois mois, pour les ressortissants de Pologne, Tchéquie, Slovaquie, Slovénie, des Etats baltes et de la Hongrie. Ce faisant, l'UE a dessiné ses futures frontières, triant entre les Etats ayant vocation à rejoindre l'Union et ceux qui resteraient dans le giron russe. Restait en suspend, la question de la Bulgarie et la Roumanie ainsi que la situation balkanique marquée alors par une grande instabilité politique.

Parmi les Européens de l'est, peu avaient les moyens de faire du tourisme en Occident mais il leur fut alors possible de s'adonner à différentes formes de business transfrontaliers. On voit ainsi se développer, en Allemagne notamment, de grands marchés improvisés où l'on peut trouver toutes sortes de marchandises venues d'Europe de l'est, en particulier de Pologne (Morokvasic, 1995). Les marchands y vendent des produits, parfois dérobés à une industrie en déroute dans leur pays d'origine, parfois importés d'ailleurs, mais dont le coût reste modique pour les Occidentaux. Ces activités engendrent de menus profits qui, grâce au différentiel de richesse, aident à subvenir aux besoins de la vie quotidienne dans des pays pour lesquels la transition économique a un coût élevé. Lors des retours, ces petits commerçants rapportent du café, des cigarettes ou des jeans qui se vendent à bon prix dans les anciennes républiques populaires.

Ce «commerce de la valise » est complété puis progressivement devancé par les migrations temporaires de travail. De faux touristes ou étudiants trouvent à se faire employer dans l'agriculture ou le bâtiment. C'est d'abord l'Allemagne puis l'Autriche, la Belgique ou la France qui attirent des travailleurs polonais, lituaniens, hongrois. Ces derniers se rendent ensuite massivement dans les pays du sud de l'Europe, en pleine croissance.

Arthur, saisonnier agricole polonais rencontré en France en 2006, raconte ainsi :

«La première fois que j'ai travaillé à l'étranger, c'était en Allemagne, en 1995, pas loin de chez moi [en Silésie], dans les asperges, pour juste un mois. Après, j'ai repris mon

\footnotetext{
${ }^{2}$ En 1991, l'espace Schengen comprend les territoires de l'Allemagne, la France, le Luxembourg, la Belgique, les Pays-Bas, l'Italie, l'Espagne et du Portugal. Il s'élargit à la Grèce en 1992, à l'Autriche en 1995 puis au Danemark, à la Suède et la Finlande en 1996. En 1999, il est intégré aux traités de l’UE.
} 
travail de tapissier chez moi puis, j'avais besoin d'argent parce que la Pologne, c'était dur. Je suis reparti avec une agence pour travailler sur un chantier, légal, encore en Allemagne, trois mois. Après, j'ai travaillé un peu en France, au Danemark... là où j'avais des amis qui peuvent m'aider à trouver du travail, mais c'est pas facile (...). En 2004, j'ai été recruté par une agence, pour cueillir les légumes en Italie. J'ai fait trois semaines et je suis parti, échappé ! On était comme des esclaves, logés dans des containers, sans douche, sans électricité. On travaillait jusqu'à la nuit, depuis le matin. Là, j'ai dit, je pars plus, maintenant je reste en Pologne. En plus, maintenant, j'ai un bébé, à la maison. Mais mon cousin est venu me chercher, pour venir en France, avec un contrat de la France, pour trois mois. C'est lui qui m'a convaincu. Alors voilà, je suis venu, pour acheter tout ce qui faut pour le bébé, refaire un peu la maison, la salle de bain. »

Dans ce genre d'expérience, les séjours légaux encadrés par des accords étatiques bilatéraux et les recrutements informels par le biais des réseaux relationnels, se succèdent sans que cela semble avoir une grande incidence pour les migrants. L'autorisation de circuler à des fins touristiques protège ces derniers des expulsions. Parallèlement, les employeurs occidentaux sont peu inquiétés lorsqu'ils embauchent des ressortissants européens sans autorisation de travail. Christophe, un agriculteur du Vaucluse, expliquait même en 2005 qu'il déclarait à la $\mathrm{MSA}^{3}$, sans que cela lui fut refusé, les revenus de ses saisonniers Slovaques à qui le marché du travail français était pourtant officiellement fermé.

C'est bien cette situation d'entre-deux, ni immigré légal ni étranger maintenu hors des frontières, qui caractérise la situation de ces migrants-touristes. Là où l'on a besoin de bras, leur présence discrète est tolérée.

Monsieur S., cultivateur de fruits et légumes dans le sud-ouest de la France raconte ainsi comment il a contribué à créer une niche d'emplois pour saisonniers agricoles polonais :

«Je suis arrivé de Pologne, j'avais 4 ans, avec ma mère, en 1954. Moi le polonais, je l'ai parlé un peu au début avec ma mère, et puis plus. C'était la Pologne communiste, on n'entretenait pas de relation, là-bas. Juste une fois, en 1981, il y a eu une ouverture. Pour lui faire plaisir, j'ai amené ma mère ; elle voulait revoir le pays alors on a passé une semaine (...). Puis en 1991, quand il y a eu les évènements et qu'on a pu voyager à nouveau, ma mère était déjà morte mais j'ai décidé de reprendre contact avec des parents, comme ça. Et là, pendant ces vacances, j'ai mon cousin qui me dit « T' as pas du travail en France pour moi ». Il voulait venir se faire un peu d'argent, parce qu'en Pologne, c'était pas la joie à cette

\footnotetext{
${ }^{3}$ Mutualité Sociale Agricole.
} 
époque. Alors je l'ai aidé. Il a travaillé pendant trois mois, pour la durée de son séjour, chez moi, puisque de toute façon, en été j'ai toujours besoin de monde (...). C'était au noir, c'est vrai. Ca s'est très bien passé, tellement bien que l'année d'après, on a fait le nécessaire pour avoir trois contrats $\mathrm{OMI}^{4}$, pour lui et deux autres de la famille (...). Et eux, ils sont venus avec quatre autres, pour travailler chez un voisin qui savait que je connaissais des Polonais et qui m'avait demandé. L'année d'après, ils [la préfecture] ont interdit les contrats OMI dans le département. Mais c'était trop tard, les gars, ils ont continué à venir ; un ici, un là. Parce que ça se passait très bien, en qu'en été ici les agriculteurs, ici, ils cherchent toujours du personnel (...). Comme ça, ça a duré pendant dix ans. Maintenant, chacun à son équipe, quoi son petit réseau, et ils ont plus besoin de moi ; ou alors ils me demandent quand ils ont besoin, de temps en temps. »

Ce que ne dit pas monsieur S., c'est qu'il ponctionne une taxe d'une centaine d'euros sur chaque mise en relation. En même temps, il intervient dans la gestion des conflits, ou comme traducteurs quand un agriculteur ne parvient pas à se faire comprendre de ses ouvriers. Ainsi, grâce à ses connexions en Pologne, monsieur S. est progressivement devenu un véritable importateur de main-d'œuvre temporaire. Lorsque, en 2004, la préfecture décide de la réouverture des contrats saisonniers, la main-d'œuvre polonaise est déjà bien implantée dans le bassin d'emploi local et la régularisation d'une partie des recrutements ne modifie que peu les pratiques développées au cours des années précédentes.

La liberté de circulation dont jouissent ces migrants leur permet, contrairement à d'autres - notamment aux Roumains et aux Bulgares - de rentrer chez eux en fin de saison sans mettre en danger de futures opportunités d'embauche dans l'espace Schengen. De cette façon, leur vie sociale et familiale se maintient dans leur pays d'origine, où ils ont presque toujours un emploi, souvent bien plus qualifié - mais moins bien rémunéré - que celui qu'ils exercent en France.

Le rôle prédominant des relations informelles dans la mise en place de ces dispositifs transnationaux apparâit clairement dans le récit de monsieur S. De la même façon, ces pratiques ont été facilitées par l'absence de contrôles de la part des services d'inspection du travail. Cette tolérance est en effet une condition indispensable à la création d'une niche d'emploi pour travailleurs illégaux.

\footnotetext{
${ }^{4}$ Contrats de travail saisonniers gérés à l'époque par l'Office de Migration Internationales français.
} 


\section{2. « Forteresse Europe » pour les autres}

Deux autres catégories de ressortissants européens ont vu, derrière le mur effondré, se dresser de hautes barricades administratives. La première concerne les pays candidats à l'adhésion à l'UE mais dont la candidature fut âprement discutée et conditionnée à de profondes évolutions ; il s'agit de la Roumanie et de la Bulgarie. La deuxième se définit par son maintien à l'extérieur de l'UE: il s'agit des Etats situés à l'est de cet espace (la Biélorussie, l'Ukraine, la Moldavie et la Russie) et de ceux de la péninsule balkanique (Serbie; Bosnie ; Croatie ; Albanie ; Macédoine). L'Europe forteresse, craignant des flux massifs, leur a fermé la porte en maintenant un régime de visa très restrictif, pour les premiers en attendant que leur situation s'améliore, pour les autres, sans limite de durée. Mais, le phénomène est connu, en matière de migration, la décision politique ne suffit pas à forcer les pratiques, elle se contente souvent de multiplier les contraintes.

Faute de pouvoir circuler librement dans l'espace Schengen, les candidats à la migration ont déployé des trésors d'inventivité pour voyager en Occident et tirer avantage de la proximité géographique de pays riches ${ }^{5}$. Dans ces pays s'est alors développée une véritable économie de la migration dans le but de contourner les restrictions officielles.

L'achat de visas touristiques, régulièrement estampillés par les ambassades européennes ou imités par des faussaires; l'usage de tour-operators que l'on quittait après l'entrée dans l'UE; les inscriptions en université dans le seul but d'obtenir un droit de séjour ou, plus sporadiquement, les passages dissimulés dans des camions ou sous des trains sont ainsi devenus les passerelles informelles de l'immigration de travail venue de l'Est (Potot, 2007).

Le récit de Radu et ses amis illustre bien ces pratiques : «En 1995, on avait juste fini l'université, et on voulait voir un peu ce qu'on peut faire à l'Ouest. On voulait voyager, travailler, voir si on peut faire quelque chose là-bas. Pendant plusieurs mois, avec S. et T., on a fait de tout, pour gagner l'argent pour partir, et aussi pour faire les papiers (...). Après 8 mois, on a eu les visas et tout ce qu'il faut, parce qu'on était sportifs, on s'est fait invités pour une compétition de sport en Allemagne (...). Quand le visa a été fini, on était en France, alors un Roumain qu'on a rencontré nous a parlé de la demande d'asile. Tu fais la demande, t'attends la réponse, quand ils te refusent tu fais le recours. Comme ça tu peux rester

\footnotetext{
${ }^{5}$ La description générale qui suit ne signifie pas que ces pays aient été également touchés par les migrations vers l'Ouest ni que celles-ci furent analysées de façon égales par la recherche. Si les travaux sur les migrations biélorusses restent lacunaires, ceux concernant la Bulgarie (Soultanova, 2005) et la Roumanie (Diminescu, 2003) témoignent de l'importance de ce phénomène dans ces pays.
} 
longtemps en France. Nous, on est resté 9 mois après le visa. Comme ça on travaillait, on vendait le journal [de rue] on mettait de coté, petit à petit, pour la Roumaine. »

Nico a eu moins de chance : «Moi, j'ai fait la demande pour un visa, mais ils ont dit non. J'ai fais deux fois, mais c'était toujours refusé. Alors j'ai décidé, avec mon copain, d'aller à pied, en se débrouillant. On est allés par la Serbie, à pied. On a marché pendant plus de 20 jours, dans la forêt, partout. Traversé la Croatie, Slovénie, jusqu'à l'Italie. Quand on est arrivé à la frontière de l'Italie, il y avait beaucoup de soldats. On avait peur qu'on tire sur nous. Avec mon copain, on a eu l'idée de passer sous un train. On s'est caché là, sous le train. Mais mon copain, il a pas tenu ; il est tombé. Clac, les deux jambes arrachées par les roues du train! On était encore en Slovénie. J'ai cru qu'il allait mourir. J'ai crié de toutes mes forces. A l'hôpital de Slovénie, ils l'ont sauvé (...). C'était en 1994. Maintenant il est dans un fauteuil roulant et on est ici [en Roumanie]. »

Face aux risques encourus, ce sont d'abord de jeunes aventuriers, pas forcément les plus pauvres, qui se sont lancés dans ces voyages vers l'ouest. Cela résultait en même temps d'une envie d'améliorer un niveau de vie en chute libre depuis la chute du communisme et de découvrir un monde moderne meilleur, fantasmé depuis de nombreuses années. Chez les jeunes, ces migrations autorisent souvent une installation dans la vie d'adulte. D'une part, la migration, avec son lot d'aventures, est perçue comme un rite initiatique vers la vie d'homme. D'autre part, elle procure souvent à ces jeunes migrants les moyens de quitter le domicile des parents et de s'installer de façon à fonder leur propre famille.

Avec les années et la multiplication des expériences d'individus partis quelques mois, voire une ou deux années, puis revenus avec de petites économies dans leur pays d'origine, la migration temporaire vers l'Ouest s'est popularisée. Les moyens de se rendre en Occident, comme les intermédiaires proposant des services en ce sens dans les pays d'origine, se multiplient au cours des années 90. Parallèlement, les migrants de retour servent souvent de relais pour diffuser des informations sur des opportunités d'embauche, des possibilités de se loger, les conditions de vie dans telle ou telle région d'Europe.

Pour beaucoup, la migration irrégulière est un challenge: elle représente un investissement important qui suppose parfois de laisser sa famille plusieurs mois et nécessite l'équivalent de plusieurs milliers d'euros. En 2002, Carmen explique que pour financer le départ de son mari vers l'Espagne où un emploi de journalier agricole l'attend, le couple a vendu quinze porcs, deux vaches et leur voiture. Cette somme finance le trajet et les (faux) documents de voyage, et rémunère également un intermédiaire supposé assurer la réussite de 
la migration. Ce dernier s'est déjà rendu dans la province espagnole et connait sur place plusieurs employeurs. Il doit aussi mettre le nouveau migrant en contact avec d'autres Roumains sur place afin de l'aider à trouver un logement. Cet intermédiaire a pour rôle d'introduire de la familiarité dans un univers inconnu pour le candidat au départ.

Les risques faiblissant avec la multiplication des expériences, la population migrante se diversifie progressivement et, concerne notamment, dès la fin des années 90, des femmes, ainsi que des personnes d'une classe d'âge plus élevée que celle des premiers aventuriers, notamment des retraités dont les pensions sont faibles.

Dans son documentaire, F.Balsamo montre comment, en Italie, des femmes venues d'Ukraine ou de Roumanie occupent à plusieurs des emplois d'assistantes de vie auprès de personnes âgées ${ }^{6}$. Assurant chacune une présence de quelques mois dans l'année, elles parviennent par ces emplois au noir très discrets puisqu'ils s'opèrent dans l'enceinte des foyers, à améliorer leur quotidien dans leur pays d'origine sans se couper durablement de leur rôle de mères et d'épouse.

Ainsi, progressivement, mêmes pour les ressortissants des pays exclus de la libre circulation, la migration temporaire de travail devient une pratique courante. Au début de la décennie 2000, de nombreuses niches d'emploi font appel à de la main-d'œuvre venue de l'Est. Bien entendu, tous les candidats au départ ne possèdent pas une carte complète des régions susceptibles d'accueillir des travailleurs européens, mais grâce à leurs connexions dans des réseaux relationnels particuliers, qui ne reposent pas seulement sur la nationalité mais aussi souvent sur la région d'origine, beaucoup partent avec une idée précise de l'endroit où ils se rendent et des possibilités qu'ils y rencontreront.

De nombreux travaux sur ces migrations montrent que celles-ci se sont en phase avec une certaine gestion informelle de l'immigration de travail en Europe. Ce fonctionnement réticulaire pousse les migrants à ne s'orienter collectivement que vers des régions où ils sont tolérés et - l'un ne va pas sans l'autre - où ils trouvent du travail. La perte d'une opportunité ou la mise en œuvre, à un moment donné, d'une répression accrue dans une région a toujours pour conséquence la recherche de nouvelles destinations et un abandon progressif de l'ancienne. Une des particularités de ces migrations tient à cette capacité à se réorienter en fonction de la conjoncture. La possibilité d'effectuer des allers-retours, même illégalement, entre des zones de travail à l'étranger et le foyer familial, cumulée à l'absence d'autorisation

\footnotetext{
${ }^{6}$ Documentaire Noapte buna - buona notte - bonne nuit, le travail de soin dans l'échange interculturel de la globalisation, par Franca Balsamo
} 
de s'installer durablement ont ainsi favorisé une circulation accrue au détriment d'une émigration durable.

Réponse au commentaire du lecteur: non, le concept de territoire circulatoire ne s'applique pas bien ici. Ce n'est pas à ces circulations «utilitaristes » que Tarrius fait référence. Quand à la «mobilité circulatoire », il me semble que comme jargon pontifiant on ne fait guère mieux.

\section{3. Vers une régularisation des migrations peu qualifiées}

Au delà de ces migrations irrégulières et peu qualifiées, des possibilités de s'expatrier en toute légalité existent depuis longtemps. Rares au début des années 90, et surtout orientées vers du personnel hautement qualifié, elles se sont multipliées à travers divers mécanismes, jusqu'à l'ouverture programmée du marché du travail européen à l'ensemble des ressortissants des Nouveaux Etats Membres (NEM), prévue au plus tard pour 2012 $2^{7}$ Deux processus conjoints ont vu le jour en Europe : d'une part, la construction et l'élargissement de l'UE ne s'envisageait pas, à moyen terme, sans une réelle liberté de circulation de tous ses travailleurs. De l'autre, tous les pays d'Europe occidentale ont progressivement admis la nécessité d'un retour à une immigration de travail. Ces deux tendances, combinées à la restructuration du monde du travail imposée par une mondialisation et à une augmentation de la xénophobie envers les populations immigrées du Sud ont joué en faveur d'une libéralisation des migrations de travail à l'échelle européenne.

Dès 1989, de nombreux accords bilatéraux ${ }^{8}$ sont mis en place pour introduire dans les pays de l'ouest une main-d'œuvre temporaire. On a cité plus haut l'accord franco-polonais de 1992 concernant les contrats saisonniers gérés par l'OMI. Cette convention rappelle celle des Gastarbeiter allemands, quotas de travailleurs étrangers, notamment à partir de 1991 des Polonais puis des Roumains et des Bulgares, «invités » à se rendre en Allemagne pour un temps et un ouvrage définis, dans la maçonnerie par exemple, avec l'obligation de quitter le pays en fin de contrat. De telles conventions, d'abord parcimonieusement conclues avec des pays du nord de l'Europe, sont aujourd'hui nombreuses.

\footnotetext{
${ }^{7}$ Seuls Chypre et Malte ont obtenu la libre circulation de leurs travailleurs dès leur adhésion ; les autres NEM dépendent de régimes particuliers négociés d'Etat à Etat, jusqu'en 2012.

${ }^{8}$ Ce type d'accords préexistait à l'est comme à l'ouest du mur de Berlin mais étaient conclus entre d'autres pays (souvent entre chacun de ces deux blocs et des pays africains ou du Golf, soit pour importer (à l'ouest) soit pour exporter (à l'est) de la main-d'œuvre.).
} 
En Espagne, les «contratos en origine » en sont une bonne illustration : depuis 2005, ils permettent de faire venir plusieurs milliers d'ouvriers agricoles au moment des périodes de récoltes. Le nombre, le profil et la (ou les) nationalité(s) des travailleurs demandés sont définis dans chaque région après négociation entre les entreprises agricoles et l'Etat. Ensuite quelques représentants des agriculteurs se rendent dans le pays d'origine et procèdent au recrutement. Selon les années et les besoins exprimés, les critères de sélection évoluent. Dans la région de Huelva où l'on produit de très grandes quantités de fraises, on a donné la préférence aux femmes, qui seraient plus délicates avec les fruits et moins susceptibles de se rebeller; si possible avec enfants en bas âge, garantie d'un retour volontaire vers le pays d'origine en fin de contrat ; et plutôt issues de régions rurales car plus endurantes au travail dans les serres (Hellio, 2009).

Affrétées par car directement depuis leur région d'origine, elles sont alors logées dans des baraquements prévus à cet effet et l'essentiel de leur vie durant ces quelques mois tourne autour de la cueillette des fraises. Ces contrats ne concernent pas que des Européennes, mais les discours racistes à l'encontre des marocains et sub-sahariens, depuis longtemps pourvoyeurs de main-d'œuvre dans l'agriculture espagnole, ont favorisé le recours à des Roumaines et des Ukrainiennes dont la culture chrétienne faciliterait l'adaptation et l'entente avec leurs employeurs espagnols. Pourtant, après que des ukrainiennes aient réclamé, comme cela était inscrit dans leur contrat, le paiement d'une partie de leurs jours chômés au début de l'année 2008, certains employeurs parlent déjà de se tourner à nouveau vers l'Afrique noire pour obtenir une main-d'œuvre plus docile (Redondo Toronjo, 2009).

Dans les pays d'origine, ce type de contrats fait l'objet de nombreux trafics, vendus jusqu'à plusieurs milliers d'euros, à crédit sur les premiers salaires. Par extension, de fausses sociétés se sont ainsi créées pour vendre à des candidats au départ de faux contrats pour l'étranger. Anticipant l'ouverture du marché du travail européen, ce type de recrutements est prisé dans la mesure où il autorise une émigration temporaire en toute légalité. Occasionnellement, ces engagements servaient également de porte d'entrée dans l'espace Schengen avant d'être abandonnés au profit d'opportunités plus lucratives trouvées sur place. Ils restent appréciés aujourd'hui car ils présentent l'assurance, pour des personnes qui n'ont pas de meilleure opportunité à l'étranger, de rentabiliser la migration.

Ces mobilités à durée déterminée ressemblent par de nombreux aspects aux migrations irrégulières. Temporaires, elles permettent à des ménages d'améliorer sensiblement leur quotidien et se concentrent dans des bassins d'emploi spécifiques. Etant gérées par des accords bipartites, elles concernent en général des secteurs, voire des zones géographiques 
que les Etats jugent se trouver dans des situations critiques. Ce sont les mêmes qui concentrent les taux les plus élevés de travail au noir: la construction, l'hôtellerierestauration, l'agriculture, les services à la personne ou le nettoyage.

Avant même que les discours politiques européens ne le reconnaissent ouvertement, la multiplication de ces accords ponctuels a permis une réouverture progressive et relativement contrôlée de l'immigration de travail. Celle-ci a souvent emboitée le pas à des pratiques illégales. La création des «contratos en origine» espagnols est par exemple explicitement intervenue pour limiter le travail au noir dans l'agriculture. Prévoyant l'ouverture du marché du travail au sein de l'UE élargie, les Etats de l'ouest ont donné la préférence aux PECO, permettant ainsi de régulariser une partie des mouvements qui avaient de toute façon vocation à perdurer.

Parallèlement à ces accords spécifiques, l'immigration temporaire de travail prend une autre forme: celle de la prestation internationale de service. Là aussi, il s'agit d'une possibilité qui préexistait mais l'adhésion des douze NEM à l'UE (10 en 2004 plus 2 en 2007) a nettement simplifié le dispositif de détachement de travailleurs au sein de l'espace européen 9 .

Dès 2004, il devient relativement simple pour une entreprise lituanienne par exemple d'envoyer une partie de ses salariés en France durant quelques mois pour participer à un grand chantier de construction. Qu'il s'agisse de la sous-traitance d'une tâche particulière ou de la mise à disposition de travailleurs intérimaires pour pallier une augmentation de l'activité, le travailleur «détaché », reste le salarié de son entreprise lituanienne et devra rentrer au siège de sa société en fin de mission. L'évolution sémantique qui change «l'immigré » en «travailleur détaché » permet ainsi de soustraire ces mouvements aux politiques migratoires pour ne les soumettre qu' aux règles du commerce international, plus souples.

Dans les secteurs qui ont le plus souvent recours au travail non déclaré des étrangers, cette formule est appréciée. Plus simple que les conventions d'importation de travailleurs, elle permet d'avoir recours à une main-d'œuvre étrangère exactement en fonction de l'évolution des besoins de l'entreprise qui s'affranchit ainsi de son rôle d'employeur pour devenir cliente d'autres sociétés. Elle n'est plus responsable de l'embauche ni de l'acheminement ou du retour des étrangers concernés. Toutes les études de terrain soulignent également les

\footnotetext{
${ }^{9}$ La directive 96/71/CE 9 encadre les prestations de services entre Etats membres et stipule que les travailleurs détachés par une entreprise de l'un de ces Etats ne sont pas soumis à la délivrance d'un permis de travail pour effectuer une tâche dans un autre pays de l'Union.
} 
débordements que ce dispositif occasionne. Les réalisateurs du film Un monde moderne témoignent du sort réservé à ces migrants sur les chantiers navals français : soumis à une législation internationale floue et difficile à faire respectée ainsi qu'à une grande opacité quant aux responsabilités de leurs employeurs, ils sont rémunérés en dessous des minima légaux, travaillent dans des conditions de sécurité particulièrement précaires et sont déplacés d'un chantier à l'autre à travers l'Europe sans aucun pouvoir sur leur propre sort $^{10}$. L'accident sur le chantier du paquebot Queen Mary, qui a fait 15 morts en 2003, serait notamment lié à la désorganisation engendrée par les interventions ponctuelles de plusieurs dizaines d'entreprises prestataires étrangères qui travaillaient sans coordination.

Malgré de nombreux litiges, tant de la part des travailleurs que des clients, ces prestations tendent aujourd'hui à se développer dans d'autres secteurs. L'agriculture et le bâtiment (Jounin, 2008), gourmands en main-d'œuvre bon marché, en sont de grands consommateurs. Leurs attraits tiennent essentiellement à la flexibilité et aux économies qu'elles offrent aux entreprises occidentales.

\section{Conclusion : vers la libre circulation des travailleurs européens}

Les prestations services comme les contrats temporaires perdront de leur intérêt aux yeux des migrants lorsqu'ils seront libres de travailler dans les mêmes conditions que les nationaux sur l'ensemble du territoire de l'UE. A l'heure actuelle, la situation européenne ressemble à un patchwork législatif. Pour tous les ressortissants des nouveaux Etats membres, une période de transition de sept années ( 2 ans +3 ans +2 ans) suivant leur date d'adhésion a été imposée avant de bénéficier de droit d'une totale liberté de travail dans l'ensemble de l'UE. Au début de chaque sous-période, chaque ancien membre de l'UE définit lui-même le régime qu'il applique à chacun des nouveaux membres. Ainsi, dès 2004, la Grande-Bretagne a ouvert la totalité de son marché du travail à tous les ressortissants des NEM. A l'inverse, la France a maintenu le sien fermé jusqu'en 2006. Seuls les secteurs dits «en tension » ont ensuite été ouverts avant une ouverture totale décrétée en 2008, pour les ressortissants des pays ayant rejoint l'UE en 2004.

Cette période de transition rend la situation européenne extrêmement complexe en théorie. Dans les faits, cette complexité est palliée, depuis plusieurs années déjà, par une grande tolérance à l'égard de l'emploi illégal de travailleurs européens. Cette façon de

\footnotetext{
${ }^{10}$ Un monde moderne réalisé par Sabrina Malek et Arnaud Soulier
} 
procéder permet en quelque sorte de tester les effets de la libre circulation des travailleurs, tout en se gardant la possibilité, à tout moment, de mettre en œuvre une politique plus répressive sur des populations ou des régions ciblées.

En marge des législations, c'est l'Europe telle qu'elle est vécue par ses citoyens qui a réellement changé au cours des deux dernières décennies. Au-delà de l'épisode du plombier polonais, les citoyens des sociétés ouest européennes rencontrent dans leur vie quotidienne des ouvriers du bâtiment roumains, des saisonniers polonais, des gardes malades ukrainiennes, des infirmières lituaniennes, des musiciens tsiganes dans le métro, etc. Cette présence ne surprend plus aujourd'hui ; elle devient le support d'échanges qui dépassent les contingences économiques et contribuent à tisser la société européenne de demain.

Documentaires :

Un monde moderne réalisé par Sabrina Malek et Arnaud Soulier

Noapte buna - buona notte - bonne nuit, le travail de soin dans l'échange interculturel de la globalisation, réalisé par Franca Balsamo

Bibliographie :

Diminescu, D. (dir.). 2003. Visibles mais peu nombreux. Les circulations migratoires roumaines. Paris, Maison des Sciences de l'Homme.

Hellio, E. 2009. Dans la province de Huelva, on importe des femmes pour exporter des fraises. Etudes rurales.

Jounin, N. 2008. Chantier interdit au public Paris, La Découverte.

Michalon, B. 2003. De la politique des Aussiedler à la circulation. Diversification des pratiques migratoires des Saxons de Transylvanie. in Visibles mais peu nombreux. Les circulations migratoires roumaines, Diminescu, D. Editions de la Maison des Sciences de l'Homme Paris, pp.65-98.

Morokvasic, M. 1995. Entre l'Est et l'Ouest, des migrations pendulaires. in Migrants. Les nouvelles mobilités en Europe, Morokvasic, M. et Rudolph, H. L'Harmattan Paris, pp.119-157.

Potot, S. 2007. Vivre à l'Est, Travailler à l'Ouest. Les routes roumaines de l'Europe. L'Harmattan.

Redondo Toronjo, D. 2009. Travailleuses étrangères dans l'horticulture intensive. De Pologne au Sénégal en passant par Huelva (Espagne). in De l'ouvrier sans papier au travailleur détaché, Morice, A. et Potot, S. à paraître.

Soultanova, R. 2005. Les migrations multiples de la population bulgare. in Balkans, C. d., La France et les migrants des Balkans: un état des lieux, Paris.

Wihtol de Wenden, C. et De Tinguy, A. 1995. L'Europe et toutes ses migrations Bruxelles, Editions complexe. 